\title{
Evidenze in tema di profilassi della nefrolitiasi ovvero come trattenere una goccia di mercurio sul polpastrello
}

Commento all'articolo: "Medical management to prevent recurrent nephrolithiasis in adults: a systematic review for an American College of Physicians Clinical Guideline”

\author{
Andrea Tasca \\ Reparto di Urologia, Ospedale S. Bortolo, Vicenza
}

La nefrolitiasi non è una malattia neoplastica e come tale viene vissuta dal paziente. Ne consegue che questi ha scarsa propensione a rispettare, una volta esauritasi l'onda emotiva che accompagna l'episodio acuto di urolitiasi, le indicazioni e le eventuali prescrizioni del medico di riferimento.

D'altro canto una recidiva della nefrolitiasi è prevedibile, nell'arco di 8 anni, in una percentuale stimata intorno al $50 \%$ dei pazienti che abbiano prodotto un calcolo renale (1). Ne deriva che oltre il 50\% dei soggetti coinvolti (non sempre le indicazioni mediche sono fruttuose) dovrebbe attenersi a stili di vita o misure terapeutiche per periodi protratti di tempo, senza avere certezza della loro reale necessità.

Questo è il motivo dominante della temporanea o parziale adesione (compliance) del paziente alle misure terapeutiche suggerite dal medico e del suo intervenuto rifiuto ad essere monitorato nel tempo (drop out), 2 aspetti messi in luce dalla maggior parte dei trials clinici (2). È pertanto indispensabile che il disegno di questi ultimi non solo definisca "l'intent-to-treat", ma preveda anche l'utilizzo di parametri che rappresentino la compliance del paziente, potenzialmente utilizzabili come indici putativi di efficacia. È il caso di 2 dei 6 trials clinici randomizzati che testano l'efficacia delle tiazidi nella profilassi della nefrolitiasi calcica, in cui vengono inclusi pazienti con ipercalciuria, vale a dire i destinatari ideali per tale forma di terapia $(3,4)$. La riduzione della calciuria funge, in questo caso, da testimonianza della compliance del paziente all'assunzione del farmaco e come rafforzativo dello "Strength of Evidence" (SOE), in un contesto in cui il numero degli eventi sintomatici non rappresenta un parametro determinante di efficacia, rispetto al gruppo di controllo. La review (5) assegna alle tiazidi un moderata SOE della riduzione del rischio 'composito' di calcolosi, trascurando di considerare quanto sopra espresso, relativamente alla compliance, oltre alla potenziale eterogeneità dei pazienti reclutati, alla diversità delle molecole impiegate (idroclorotiazide, clortalidone, indapamide) e delle relative posologie. La diagnostica per immagini, affidata a ecografia e radiografia in bianco dell'addome, rappresenta, inoltre, nei lavori citati, l'evidenza più concreta dell'efficacia o fallimento della profilassi antilitiasica. L'affidabilità di tali indagini è, tuttavia, tutt'altro che assoluta tenuto conto del fatto che la loro sensibilità, in caso di utilizzo combinato, supera di poco il $75 \%$, mentre quella della TAC in bianco, anche a basso dosaggio, raggiunge il $97 \%(6,7)$. Ne deriva che tale indagine, per la sua affidabilità e per la minima esposizione radiogena, dovrebbe essere imperativamente utilizzata nei futuri trials clinici.

Il citrato si conferma, nella rassegna presentata, un agente efficace nella prevenzione delle recidive anche nel paziente che non presenti un'ipocitraturia e ciò assimila gli studi condotti in questo contesto a quelli in precedenza citati, relativi alle tiazidi. Sono stati, tuttavia, esclusi dall'analisi 2 trials in cui il citrato di Na e K si rivela inefficace nella profilassi delle recidive litiasiche (8, 9). L'evidenza complessiva sembrerebbe, pertanto, indicare che il citrato di $\mathrm{K} \mathrm{e} \mathrm{Mg}$ sia preferibile, rispetto al sale sodico, per la profilassi antilitiasica, contrariamente a quanto espresso dagli Autori della review (5).

I regimi orientati alla prevenzione delle recidive litiasiche si fondano in larga misura sull'incrementato apporto di liquidi e sulle manipolazioni dietetiche. Uno dei 2 trials relativi al primo di questi regimi, citati nella review (5), documenta l'efficacia dell'H20 nella prevenzione delle recidive (10). Il limite del lavoro, al di là delle impurità statistiche, è relativo alla mancata definizione delle caratteristiche dell'H20 utilizzata e allo schema di assunzione dei liquidi. Tralasciando la storica disputa sulla reale utilità delle acque 'tenere', va considerata l'evenienza di potenziali picchi di sovrasaturazione salina nel liquido tubulare del rene. La conseguente precipitazione di cristalli, può infatti intervenire, a dispetto di un consistente apporto quotidiano di liquidi, qualora questi non vengano assunti omogeneamente nell'arco delle 24 ore (11).

Le manipolazioni dietetiche sono difficili da valutare nel lungo termine. È richiesta un'intensa motivazione da parte del paziente che lo renda disponibile ad essere valutato con periodicità sul versante biochimico e della saturazione delle urine, oltre che su quello della diagnostica per immagini e una devozione del medico tale da avvicinare questi, quanto più possibile, al paziente. I lavori citati, in quest'ambito, nella review (5) sono orientati alla prevenzione delle recidive litiasiche modificando più componenti della dieta, un contesto in cui è difficile valutare la compliance del paziente, senza indagare questi con frequenti analisi meta- 
boliche, al di là dell'utilizzo dei questionari. Lo studio ideale dovrebbe prevedere la modifica di singoli componenti della dieta, estendersi per periodi protratti di tempo (5-7 anni) ed includere periodici controlli biochimici e un puntuale studio radiologico. Dalla review emergono complessivamente, delle evidenze cedevoli che sono in parte dovute non solo alle caratteristiche della malattia litiasica, il cui andamento è spesso ondivago ed alla compliance del paziente, come già sottolineato, ma anche al disegno non sempre puntuale degli studi.

Un primo passo da compiere riguarda, a mio avviso, l'affrancamento culturale del medico che tuttora considera la nefrolitiasi come una malattia d'organo o d'apparato, piuttosto che d'organismo. Andrà poi considerato uno studio epidemiologico su vasta scala che analizzi longitudinalmente le modificazioni del profilo clinico del paziente litiasico, orientato in particolare sulla funzione renale e sull'osso. Seguirà un'accurata informazione del paziente che lo renda incline a considerare la malattia litiasica come un evento non necessariamente episodico e organo confinato.

Dichiarazione di conflitto di interesse: L'Autore dichiara di non avere conflitto di interessi.

Contributi economici agli Autori: L'Autore dichiara di non avere ricevuto sponsorizzazioni economiche per la preparazione dell'articolo.

Indirizzo dell'Autore:

Dr. Andrea Tasca

Reparto di Urologia

Ospedale S. Bortolo

Via Rodolfi 37

36100 Vicenza

andrea.tasca@ulssvicenza.it

\section{Bibliografia}

1. Lijunghall S, Danielson G. A prospective study of renal stone recurrence. Br J Urol 1984; 56: 122-4.

2. van Drongelen J, Kiemeney LA, Debruyne FM, de la Rosette JJ. Impact of urometabolic evaluation on prevention of urolithiasis: a retrospective study. Urology 1998; 52: 384-91.

3. Ohkawa M, Tokunaga S, Nakashima, et al. Thiazide treatment for calcium urolithiasis in patients with idiopathic hypercalciuria. Br J Urol 1992; 69: 571-6.

4. Borghi L, Meschi T, Guerra A, Novarini A. Randomized prospective study of a non thiazide diuretic, indapamide in preventing calcium stones. J Cardiovasc Pharmacol1993; 22(Suppl): S78-86.

5. Fink HA, Wilt TJ, Eidman KE, et al. Medical management to prevent recurrent nephrolithiasis in adults: a systematic review for an American College of Physicians Clinical Guideline. Ann Intern Med 2013; 158(7): 535-43.

6. Ray AA, Ghiculete D, Pace KT, et al. Limitations to ultrasound in the detection and measurement of urinary tract calculi. Urology 2010; 76(2): 295-300.

7. Catalano O, Lobianco R, Altei F, Siani A. Suspected ureteral colic: primary helical CT versus selective helical CT after unenhanced radiography and sonography. AJR Am J Roentgenol 2002;178(2): 379-87.

8. Jendle-Benden C, Tiselius HG. Long-term follow-up of stone formers treated with a low dose sodium potassium citrate. Scand J Urol 1994; 151: 5.

9. Abdulhadi MH, Hall PM, Streem SB. Can citrate therapy prevent nephrolithiasis? Urology 1993; 41: 221-4.

10. Borghi L, Meschi T, Amato F, Briganti A, Novarini A, Giannini A. Urinary volume, water and recurrences in idiopathic calcium nephrolithiasis: a 5-year randomized prospective study. J Urol 1996; 155: 839-43.

11. Tiselis HG. Who forms stones and why? Eur Urol 2011; Suppl. 10(5): 408-14. 